\section{Venetoclax plus rituximab or obinutuzumab after allogeneic hematopoietic stem cell transplantation in chronic lymphocytic leukemia}

For several decades, allogeneic hematopoietic stem cell transplantation (alloHCT) has been a therapeutic option in patients with chronic lymphocytic leukemia (CLL) with high-risk features such as $\operatorname{del}(17 \mathrm{p})$ and/or TP53 mutations, complex aberrant karyotype, or chemotherapy-resistant disease. With the advent of new targeted agents, like ibrutinib, idelalisib, or venetoclax, an increasing number of patients with CLL is now managed without undergoing alloHCT. ${ }^{1,2}$ While alloHCT has curative potential, many patients with CLL are not eligible for transplant due to age and co-existing conditions, as the procedure can entail con- siderable treatment-related morbidity and mortality. Moreover, up to $50 \%$ of patients may experience relapsed disease after transplantation. ${ }^{3,4}$

Venetoclax, a BH3-mimetic that inhibits Bcl-2, has shown profound efficacy in patients with CLL. It is currently approved for treatment of patients with relapsed/refractory CLL, including patients with high-risk genetic abnormalities like del(17p) or TP53 mutations. ${ }^{5}$ Several clinical trials are investigating its use in first-line therapy, as monotherapy, or in combination with other drugs. ${ }^{6}$ However, data on patients with relapsed disease after alloHCT are limited, as most clinical trials have excluded this subset of patients from enrollment.

Here, we report clinical data from four patients with CLL (Table 1) who were successfully treated with venetoclax for

Table 1. Patients' characteristics.

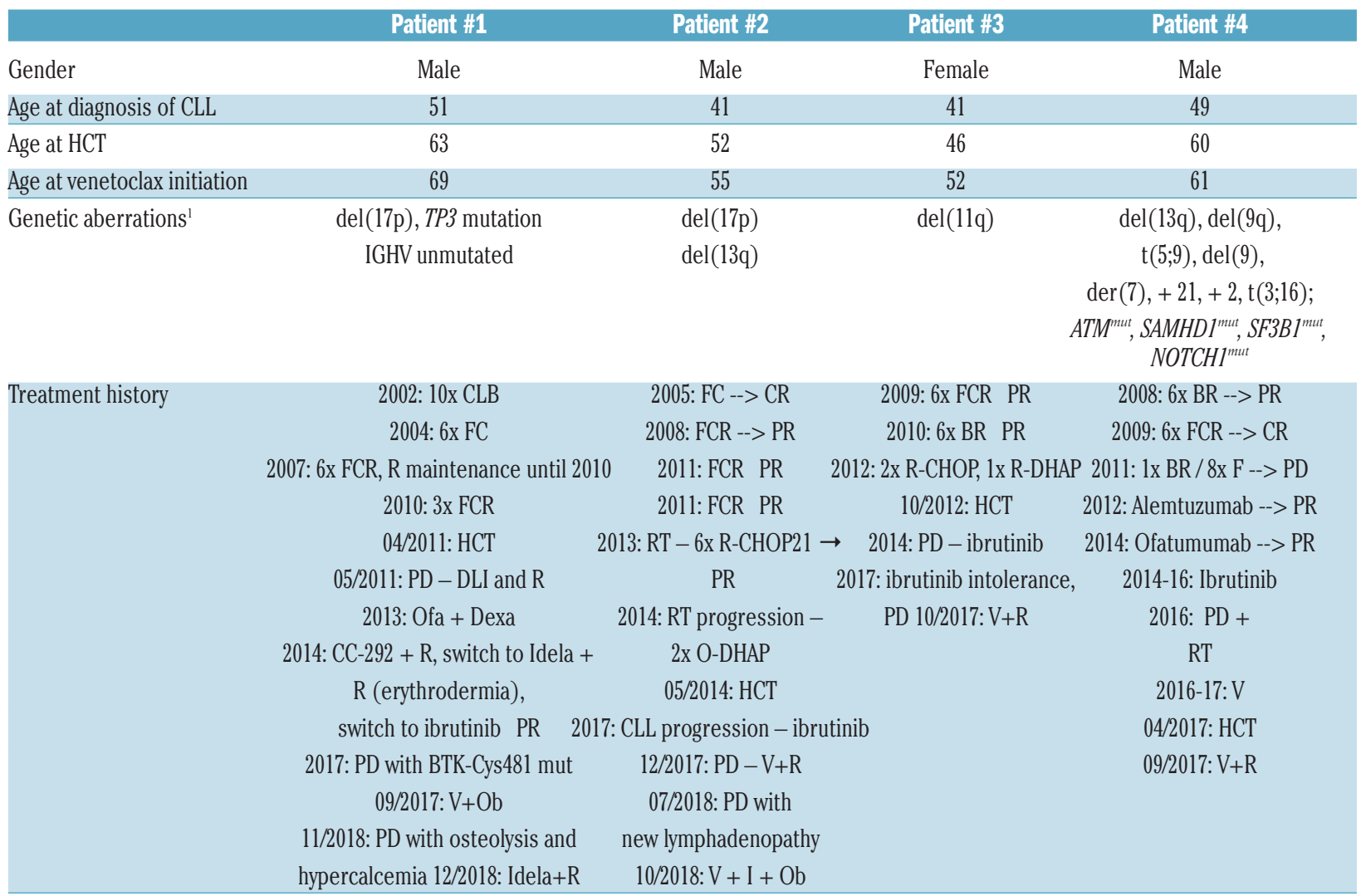

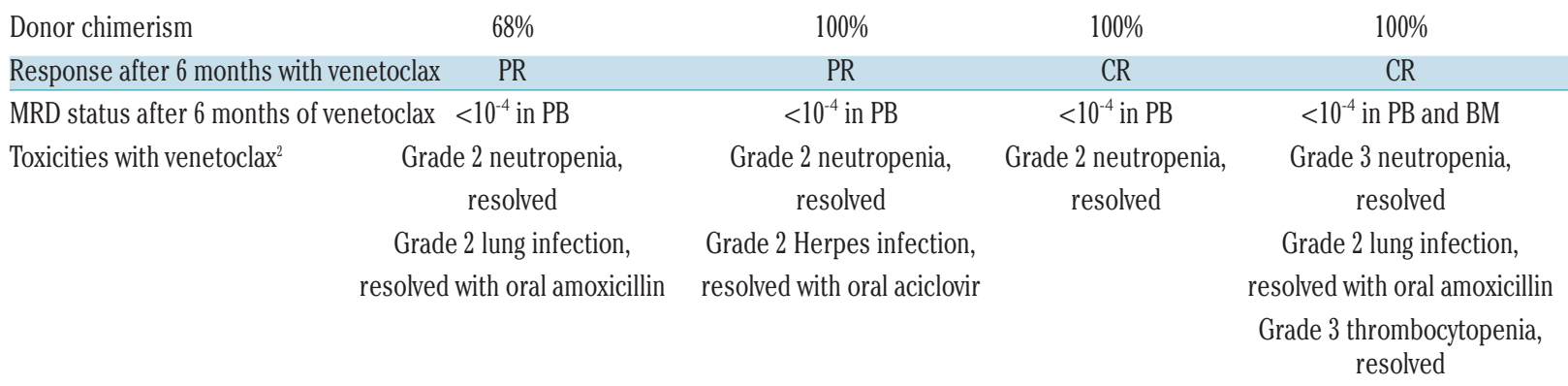

CLL: chronic lymphocytic leukemia; RT: Richter's transformation; MRD: minimal residual disease; CLB: chlorambucil; FC: fludarabine + cyclophosphamide; FCR: FC + rituximab; R: rituximab; BR: bedamustine + rituximab; HCT: hematopoietic stem cell transplantation; DLI: donor lymphocyte infusion; Ofa: ofatumumab; Dexa: dexamethasone; Idela: idelalisib; I: ibrutinib; CR: complete remission; PR: partial response; PD: progressive disease; mut: mutation; R-CHOP: rituximab, cyclophosphamide, hydroxydoxorubicine, oncovine, prednisolone; DHAP: dexamethasone + high-dose cytarabine + cisplatin; V: venetoclax; Ob: obinutuzumab; PB: peripheral blood; BM: bone marrow. ${ }^{1}$ Parameters are partially incomplete as cyto-/molecular genetic analyses were not completely available at the time of first-line treatment. ${ }^{2} \mathrm{Grading}$ according to National Cancer Institute Common Terminology Criteria for Adverse Events v.4. ${ }^{3}$ Last follow-up visit in December 2018 
relapsed disease after alloHCT. These constitute all patients who received venetoclax after alloHCT at our center.

The first patient is a 70-year old man who was diagnosed with CLL in 1999. Initial adverse genetic aberrations were $\operatorname{del}(17 p)$ and TP53 mutation. Prior to alloHCT, the patient received treatment with chlorambucil, fludarabine plus cyclophosphamide (FC), FC plus rituximab (FCR) and rituximab maintenance therapy (Table 1). In April 2011, he underwent HLA-matched related donor alloHCT after conditioning with FC. He experienced only mild grade 1 acute graft-versus-host disease (GvHD) of the skin and small joints that were well controlled by cyclosporine (CSA) and mycophenolate mofetil (MMF). One month after transplantation, the patient presented a fourth relapse, which was treated with rituximab and donor lymphocyte infusions (DLI). Subsequent relapses were treated with ofatumumab plus dexamethasone, idelalisib plus rituximab, and ibrutinib, respectively. The patient achieved a partial remission (PR) with ibrutinib monotherapy until 2017, when a BTK-Cys481 mutation was detected and the patient showed progressive lymphadenopathy. The patient received obinutuzumab infusions and started venetoclax ramp up in September 2017. He showed a PR due to residual lymph nodes with minimal residual disease (MRD) negativity in peripheral blood (PB), assessed by flow cytometry with a $10^{-4}$ threshold. He continued daily $400 \mathrm{mg}$ venetoclax until November 2018 when new osteolysis as well as hypercalcemia were observed, which were related to disease progression. Idelalisib and rituximab were subsequently re-introduced, given that this treatment had previously only been discontinued because of erythrodermia of unclear origin. At data cutoff, no response assessment was possible due to the short drug administration time.

The second patient is a 56-year old man who was diagnosed with CLL in 2003. Adverse genetic aberrations were $\operatorname{del}(17 p)$. The patient received 6 cycles of FC as his first-line therapy in 2005 and achieved a complete remission (CR). Relapses were treated with 6 cycles of FCR in 2008 and 2011, and Richter's transformation (RT) was treated with 6 cycles of R-CHOP21 in 2013. In 2014, the patient showed progression of RT and was prepared for alloHCT. For remission induction, two cycles of ofatumumab plus DHAP were administered followed by weekly ofatumum$\mathrm{ab}$ administrations until an HLA-matched related donor alloHCT was performed in May 2014 after fludarabine/busulfan/ofatumumab conditioning. $\mathrm{He}$ received GvHD prophylaxis with CSA and MMF under which no signs of acute GvHD occurred; however, suspected mild intestinal and cutaneous GvHD was observed in 2016 due to intermittent diarrhea and rash. These resolved with short-term steroids and tacrolimus. The patient showed a CR of RT, however, due to progressive CLL with $\operatorname{del}(17 p)$, ibrutinib was introduced in 2017. After 8 months of ibrutinib treatment, the patient showed new cutaneous lesions as well as a new pre-sternal positron emission tomography (PET)-positive lymph node; this was biopsied and revealed infiltration by CLL. Venetoclax was introduced after ramp-up to $400 \mathrm{mg}$ daily in December 2017 together with monthly rituximab infusions. Response assessment in June 2018 showed a mixed response with an enlarged, PET-positive pre-sternal lesion, despite peripheral MRD blood negativity. Irradiation of the lesion was initiated, followed by ongoing DLI. Remission control via PETcomputed tomography (CT) initially showed a partial response with complete resolution of the irradiated presternal lesion; however, due to recurrent lymph adenopathy, treatment was extended in October 2018 with the addition of ibrutinib and obinutuzumab to venetoclax. Data cutoff was too early for complete response assess-
Cellular Immune Status

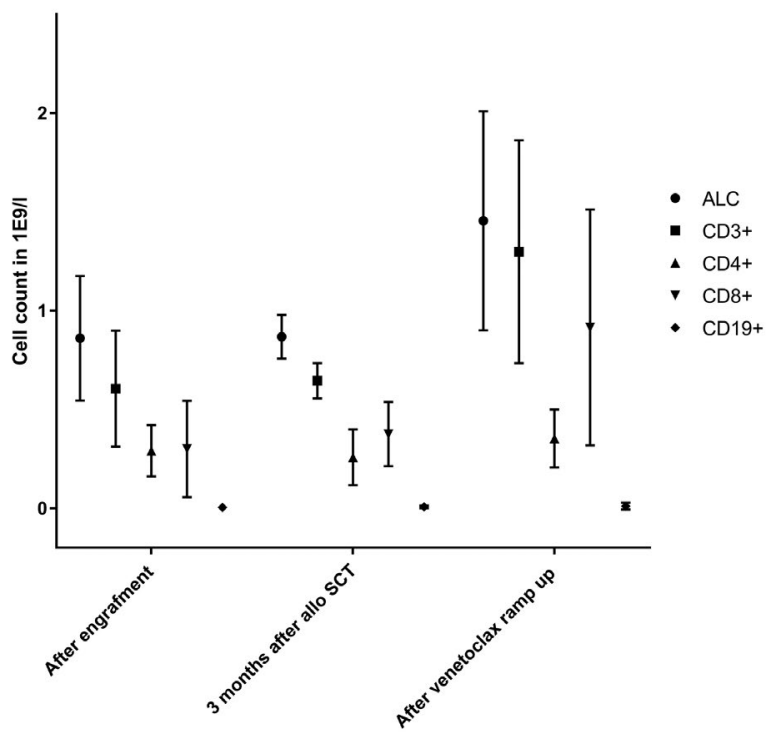

Figure 1. Cellular immune status during treatment. Cellular immune status of all four patients was assessed by flow cytometry after engraftment, 3 months after transplantation, and after completion of venetoclax ramp up (i.e. approx. 5 weeks after first dose). allo SCT: allogeneic stem cell transplantation; ALC: absolute lymphocyte count. Error bars represent standard deviation.

ment; however, so far the patient has shown stable disease under the triple combination.

The third patient is a 52-year old female who was diagnosed with small lymphocytic lymphoma in 2007 . She presented with a del(11q). The patient was treated with FCR, bendamustine plus rituximab (BR), R-CHOP and R-DHAP. In October 2012, an alloHCT with an unrelated mismatched donor was performed after treosulfan/fludarabine conditioning. One month after transplantation, the patient developed grade 2 cutaneous and grade 1 hepatic GvHD that was managed with CSA and MMF. Two years after transplantation, a progression was treated with ibrutinib, which had to be discontinued despite a CR due to repetitive complications including arrythmia and diarrhea, upon which a rapid nodal progression occurred. The patient was on low-dose prednisolone $4 \mathrm{mg}$ and ruxolitinib $15 \mathrm{mg}$ daily due to chronic steroid-dependent cutaneous GvHD. In October 2017, venetoclax ramp-up to $400 \mathrm{mg}$ daily plus monthly rituximab were initiated. The most recent remission control in December 2018 showed no residual lymphadenopathy with MRD-negativity in the peripheral blood.

The fourth patient is a 61-year old man who was diagnosed with CLL at the age of 49 years. He had received treatment with F, BR, FCR, later followed by alemtuzumab and ofatumumab. The patient developed a complex karyotype with several unfavorable mutations (Table 1). After developing erythroderma under idelalisib treatment, he was treated with ibrutinib, which was discontinued in 2016 due to atrial fibrillation, orchitis and, finally, disease transformation. Venetoclax was administered daily for 3 months at a dose of $1200 \mathrm{mg}$, as the patient initially did not respond to $400 \mathrm{mg}$; the patient showed a mixed response with cervical and axillary lymph nodes completely regressing, while inguinal lymph nodes were progressing. After conditioning with fludarabine/busulfan, the patient underwent alloHCT in April 2017 and showed a complete remis- 
sion 2 months later. The patient developed grade 1-2 intestinal GvHD and grade 3 ocular GvHD, which were treated with CSA, MMF and steroids. At 4 months, axillary lymph nodes and inguinal lymph nodes were increasing in size. Biopsies were taken from the inguinal lymph nodes with the strongest fluorodeoxyglucose (FDG) PET-CT scan signal. However, immunohistochemistry revealed mature CLL cells without RT. Venetoclax treatment was initiated again, up to a maximum dose of $400 \mathrm{mg}$ plus monthly rituximab. A CR was documented 8 weeks after venetoclax initiation. Response control after 6 months of venetoclax confirmed a sustained CR via PET-CT as well as MRD-negativity in bone marrow. Response was maintained at the latest follow up in December 2018.

As novel targeted agents have shown considerable activity in high-risk and chemorefractory CLL with less treatment-associated morbidity, in recent years, the use of alloHCT in CLL management has been decreasing. However, for patients who fail multiple lines of therapy and have high-risk genetic profiles, alloHCT often remains the only viable clinical option. ${ }^{7}$ Moreover, patients who underwent alloHCT before novel agents became available might still experience disease progression and require therapy. Very limited data are available for this group of patients, as most clinical trials have excluded patients with prior alloHCT. There are two published reports with selected cases on ibrutinib use after alloHCT for CLL. ${ }^{8,9}$ Both studies showed that BTK inhibition was effective in 5 and 38 patients, respectively, after alloHCT, even after second transplantation. Overall response rate was $100 \%$ and $88 \%$, respectively, and 2 of the $5(40 \%)$ and $4(11 \%)$ of the 38 patients had reported MRD negativity. Similar outcomes were observed in a retrospective analysis of 5 patients treated with ibrutinib after alloHCT. ${ }^{10}$

In our small series of patients, venetoclax demonstrated activity in highly pre-treated patients, including one patient with RT. Except for Patient \#2, who required additional irradiation of nodal lesions, three patients showed nodal response, and none of the four patients had detectable MRD. Venetoclax was generally well tolerated and no severe adverse events were observed at a dose of $400 \mathrm{mg}$. However, caution is warranted as patients after alloHCT regularly have poor immune status and are sensitive to infections which might be potentially aggravated by venetoclax. ${ }^{11}$ While T-cell activity seemed to be completely restored during venetoclax therapy, all patients in our series showed ongoing CD19 deficiency (Figure 1), most probably as result of anti-CD20 therapy. It is currently not clear how treatment with venetoclax might affect GvHD. While ibrutinib has shown efficacy in chronic, steroid-refractory $\mathrm{GvHD},{ }^{12}$ and is approved for treatment of chronic GvHD in the US, recent preclinical data demonstrated that ABT-737 (a Bcl-2 and Bcl-xl inhibitor) alleviates GvHD by shifting the balance towards regulatory $\mathrm{T}$ cells (Tregs) in a murine model $1 .{ }^{13}$ Since venetoclax spares $\mathrm{Bcl}-\mathrm{xl}$ inhibition, it remains unknown if venetoclax is also able to shift the balance towards Tregs. Taking this into account, one might speculate that venetoclax at least does not enhance a graftversus-leukemia effect, although further studies are necessary to better understand the effect of venetoclax on immunological response. No patient in our cohort showed exacerbation of GvHD after venetoclax initiation. It should be noted that all patients were treated with a combination of venetoclax plus an anti-CD20 antibody. From our experience, as well as according to the latest trial results, addition of rituximab or obinutuzumab considerably increases response to therapy as compared to venetoclax monotherapy. ${ }^{14,15}$ Hence, a combined approach in heavily pre-treated patients, as in this case series, appears to be justified.
In conclusion, our case series indicates that treatment with venetoclax is feasible and effective in patients with CLL and relapsed disease after alloHCT. Larger sets of patients and extended follow up are necessary to further investigate the rate and quality of remissions and long-term complications.

Othman Al-Sawaf, Carmen Diana Herling, Udo Holtick, Christoph Scheid, Paula Cramer, Stephanie Sasse, Bastian von Tresckow, Armin Tuchscherer, Kirsten Fischer, Barbara Eichhorst, Michael Hallek and Lukas P. Frenzel

University of Cologne, Department I of Internal Medicine, Center for Integrated Oncology Aachen-Bonn-Cologne-Duesseldorf, Germany

Acknowledgments: we thank the patients and their families for the trust and confidence they have placed in us as well as the team of the outpatient oncology and transplant unit at the University Hospital of Cologne

Correspondence: LUKAS FRENZEL.

lukas.frenzel@uk-koeln.de

doi:10.3324/haematol.2018.212837

Information on authorship, contributions, and financial \& other disclosures was provided by the authors and is available with the online version of this article at WWw. haematologica.org.

\section{References}

1. Gribben JG. How and when I do allogeneic transplant in CLL. Blood. 2018;132(1):31-39

2. Hallek M, Shanafelt TD, Eichhorst B. Chronic lymphocytic leukaemia. Lancet. 2018;391(10129):1524-1537.

3. Dreger P, Schetelig J, Andersen N, et al. Managing high-risk CLL during transition to a new treatment era: stem cell transplantation or novel agents? Blood. 2014;124(26):3841-3849.

4. Kramer I, Stilgenbauer S, Dietrich S, et al. Allogeneic hematopoietic cell transplantation for high-risk CLL: 10-year follow-up of the GCLLSG CLL3X trial. Blood. 2017;130(12):1477-1480.

5. Stilgenbauer S, Eichhorst B, Schetelig J, et al. Venetoclax for Patients With Chronic Lymphocytic Leukemia With 17p Deletion: Results From the Full Population of a Phase II Pivotal Trial. J Clin Oncol. 2018;36(19):1973-1980.

6. Seymour JF, Kipps TJ, Eichhorst B, et al. Venetoclax-Rituximab in Relapsed or Refractory Chronic Lymphocytic Leukemia. N Engl J Med. 2018;378(12):1107-1120.

7. Dreger P, Ghia P, Schetelig J, et al. High-risk chronic lymphocytic leukemia in the era of pathway inhibitors: integrating molecular and cellular therapies. Blood. 2018;132(9):892-902.

8. Link CS, Teipel R, Heidenreich F, et al. Durable responses to ibrutinib in patients with relapsed CLL after allogeneic stem cell transplantation. Bone Marrow Transplant. 2016;51(6):793-798.

9. Ryan CE, Sahaf B, Logan AC, et al. Ibrutinib efficacy and tolerability in patients with relapsed chronic lymphocytic leukemia following allogeneic HCT. Blood. 2016;128(25):2899-2908.

10. Rozovski U, Benjamini O, Jain P, et al. Outcomes of Patients With Chronic Lymphocytic Leukemia and Richter's Transformation After Transplantation Failure. J Clin Oncol. 2015;33(14):1557-1563.

11. Davids MS, Hallek M, Wierda W, et al. Comprehensive Safety Analysis of Venetoclax Monotherapy for Patients with Relapsed/Refractory Chronic Lymphocytic Leukemia. Clin Cancer Res. 2018;24(18):4371-4379.

12. Miklos D, Cutler CS, Arora M, et al. Ibrutinib for chronic graft-versus-host disease after failure of prior therapy. Blood 2017;130(21):2243-2250.

13. Gabriel SS, Bon N, Chen J, et al. Distinctive Expression of Bcl-2 Factors in Regulatory T Cells Determines a Pharmacological Target to Induce Immunological Tolerance. Front Immunol. 2016;7:73.

14. Fischer K, Al-Sawaf O, Fink AM, et al. Venetoclax and obinutuzumab in chronic lymphocytic leukemia. Blood. 2017;129(19):2702-2705.

15. Seymour JF, Ma S, Brander DM, et al. Venetoclax plus rituximab in relapsed or refractory chronic lymphocytic leukaemia: a phase $1 \mathrm{~b}$ study. Lancet Oncol. 2017;18(2):230-240. 